\title{
The genetics of hepatitis $C$ virus underlie its ability to escape humoral immunity
}

\author{
Jay K. Kolls ${ }^{1}$ and Gyongyi Szabo ${ }^{2}$ \\ 'University of Pittsburgh School of Medicine, Department of Pediatrics, Children's Hospital of Pittsburgh of UPMC, Pittsburgh, Pennsylvania, USA. ${ }^{2}$ Department of Medicine, \\ University of Massachusetts Medical School, Worcester, Massachusetts, USA.
}

\begin{abstract}
Hepatitis C virus (HCV) is a leading cause of chronic liver disease, and efforts to develop therapeutic vaccine strategies have been limited by immune escape due to HCV variants that are resistant to current vaccines or HCV variants that rapidly acquire new resistance-conferring mutations. Recently, the crystal structure of the viral envelope protein E2 region was resolved as well as how E2 docks to the host CD81 protein; therefore, antibodies that block this interaction should prevent viral entry into host cells. In this issue of the $J C l$, Bailey and colleagues show that immune escape of HCV can occur by naturally occurring polymorphisms in E2 that are distinct from those at mapped sites of antibody binding. These data reveal alternative mechanisms of resistance that need to be considered in both natural viral escape as well as in rationale vaccine design against HCV.
\end{abstract}

\section{Hepatitis C virus: treatment options and cost}

Hepatitis $\mathrm{C}$ virus (HCV) is an enveloped positive strand RNA virus that infects up to 150 million individuals worldwide. HCVinfected individuals are at increased risk of developing cirrhosis and hepatocellular carcinoma. The economic burden of HCV in the US alone is estimated to be $\$ 6.5$ billion annually with increasing annual costs projected through at least 2024 (1). Treatment strategies have centered on antiviral drugs, such as ribavirin and type I interferons (2); however, HCV exists as a quasispecies that includes a variety of genotypes that are differentially susceptible to these regimens. Currently, 6 genotypes are recognized, with varying numbers of subspecies within each of these 6 clades. Recently, the drug sofosbuvir, a nonstructural protein $5 \mathrm{~B}$ (NS5B) RNA polymerase inhibitor, was approved for use in combination with ribavirin to treat HCV genotypes 2 and 3. Sofosbuvir is also approved for use in combination with ribavirin and pegylated interferon for use against HCV genotypes 2 and 4. These regimens have been shown to achieve up to $90 \%$ response rates in patients, particularly in those infected with HCV genotype 1 or 2 (2). The newest FDA-approved oral regimen, which consists of ledipasvir (a NS5A inhibitor) and sofosbuvir, achieved up to $99 \%$ cure rate in noncirrhotic patients infected with HCV genotype 1 after 12 weeks of treatment (3).

\section{The search for an effective vaccine}

Although much progress has been made in treating HCV, the development of a preventative vaccine remains a major goal in the field. The basis for HCV vaccination is that patients will develop neutralizing antibodies that target HCV viral envelope proteins, thereby inhibiting viral entry into host cells. Multiple monoclonal antibodies with demonstrated ability to neutralize viral attachment or entry into host

Related Article: p. 437

Conflict of interest: Jay K. Kolls has received research grants from Constellation Pharmaceuticals and Amgen in areas unrelated to this work

Reference information: J Clin Invest. 2015;125(1):97-98. doi:10.1172/JCI79424. 
mapped to a region in E2 that encompasses amino acids 416-560, which span the front layer, the CD81-binding motif, and the central $\beta$-sheet region of the envelope. Specifically, polymorphisms in amino acids 431, 442, and 560 resulted in limited resistance to the antibody panel; however, mutations in the central $\beta$-sheet (amino acids 526-569) conferred broad resistance to the antibodies tested. Analysis of the envelope proteins expressed by susceptible clones and the envelope proteins expressed by resistant HCVppexpressing clones revealed 7 shared variant sites. Introduction of resistance-associated mutations into susceptible HCV envelops conferred neutralization resistance, and introduction of susceptibility-associated mutations in resistant envelopes resulted in sensitivity to antibody neutralization. Bailey and colleagues (6) identified three critical mutations (I538V, Q546L, and T563V) that mediate resistance to both monoclonal antibodies and polyclonal antibodies. Importantly, these mutations were not in areas of the envelope that mapped to monoclonal antibody-binding sites. Although some of these mutations may confer some negative effect on viral fitness, the identification of these mutations may also explain HCV immune escape. The exact mechanisms of resistance conferred by these mutations are currently not known but may involve alterations in protein folding or glycosylation, which could mask epitopes or create steric hindrance and thereby reduce antibody binding. In addition, receptor-independent transfer of replication-competent HCV RNA by exosomes may also undermine the efficacy of antibody-mediated protection (8). Future studies will need to focus on how these different mutations confer resistance and the overall contribution of exosome-mediated viral entry in immune escape.

\section{Conclusions and future directions}

The level of polymorphisms within the $\mathrm{HCV}$ envelope that confer antibody resistance makes strategies to achieve thera- peutic vaccination or to develop broadly effective therapeutic monoclonal antibodies more difficult. Based on the propensity of immune escape, it is unlikely that a vaccine directed against just one region of the HCV envelope will prove effective. This type of multivalent approach to vaccine generation would be similar to the approach used for pneumococcal immunization, which provides protection against multiple pneumococcal serotypes but does not protect against all strains. Potential multivalent $\mathrm{HCV}$ vaccines would consist of the most common viral E1E2 variants based on viral genomics. A multivalent approach to HCV immunization will be costly, based on both production costs as well as chemistry, manufacturing, and controls costs, which would be required to ensure the safety of each vaccine component. The work by Baily et al. (6) clearly demonstrates that monoclonal antibody approaches may not be feasible to provide broad HCV protection, as even bispecific antibodies would likely encounter immune escape. The study by Bailey and colleagues does provide a platform through which new antibodies can be screened. This type of cell-based platform to test the efficacy of vaccine candidates in their ability to neutralize a variety of HCVpp will clearly benefit future clinical development. We are currently in an era in which treatment for HCV-infected individuals has reached new heights in therapeutic efficacy. Further work on identifying envelope variants will hopefully help realize the potential of an $\mathrm{HCV}$ vaccine.

\section{Acknowledgments}

G. Szabo acknowledges support from NIH grant R37AA014372, and J.K. Kolls was supported by NIH grant R37HL079142.

Address correspondence to: Jay K. Kolls, Children's Hospital of Pittsburgh of UPMC, RRB 8130, 4401 Penn Ave., Pittsburgh, Pennsylvania 152224, USA. Phone: 412.692.7710; E-mail: jay.kolls@chp.edu. Or to: Gyongyi Szabo, UMass Memorial Medical Center, 55 N. Lake Ave., Worces- ter, Massachusetts 01655, USA. Phone: 508.856.5275; E-mail: Gyongyi.Szabo@ umassmed.edu.

1. Razavi $\mathrm{H}$, et al. Chronic hepatitis $\mathrm{C}$ virus (HCV) disease burden and cost in the United States. Hepatology. 2013;57(6):2164-2170.

2. [No authors listed]. A SPECIAL MEETING REVIEW EDITION: Advances in the Treatment of Hepatitis C Virus Infection from The Liver Meeting 2013: The 64th Annual Meeting of the American Association for the Study of Liver Diseases November 1-5, 2013 - Washington DC Special Reporting on: Simeprevir plus Sofosbuvir with or without Ribavirin Produces High SVR Rates in Genotype 1 HCV Infection - Novel Interferon- and Ribavirin-Free Regimen Results in SVR12 Rates of Over 90\% in HCV Genotype $1 \mathrm{~b}$ Infection. Studies Confirm Efficacy of Adjunctive Simeprevir in Difficult-to-Treat HCV Genotype 1 Subpopulations• All-Oral Therapy with Sofosbuvir Plus Ribavirin Produces High SVR Rates in Patients Coinfected with HCV and HIV · Faldaprevir Combined with Pegylated Interferon and Ribavirin Demonstrates High Efficacy in DifficuIt-to-Treat HCV Infection. Once Daily Sofosbuvir/Ledipasvir Combination Elicits Rapid Decline in HCV RNAPLUS Meeting Abstract Summaries With Expert Commentary by: Ira M. Jacobson, MD Weill Cornell Medical College, New York, New York. Gastroenterol Hepatol (N Y). 2014;10 (1 suppl 1):1-19.

3. Afdhal N, et al. Ledipasvir and sofosbuvir for untreated HCV genotype 1 infection. N EnglJ Med. 2014;370(20):1889-1898.

4. Sabo MC, et al. Neutralizing monoclonal antibodies against hepatitis $\mathrm{C}$ virus $\mathrm{E} 2$ protein bind discontinuous epitopes and inhibit infection at a postattachment step. J Virol. 2011;85(14):7005-7019.

5 . Keck ZY, et al. Non-random escape pathways from a broadly neutralizing human monoclonal antibody map to a highly conserved region on the hepatitis C virus E2 glycoprotein encompassing amino acids 412-423. PLoS Pathog. 2014;10(8):e1004297.

6. Bailey JR, et al. Naturally selected hepatitis $\mathrm{C}$ virus polymorphisms confer broad neutralizing antibody resistance. J Clin Invest. 2015;125(1):437-447.

7. Osburn WO, et al. Clearance of hepatitis C infection is associated with the early appearance of broad neutralizing antibody responses. Hepatology. 2014;59(6):2140-2151.

8. Bukong TN, Momen-Heravi F, Kodys K, Bala S, Szabo G. Exosomes from hepatitis C infected patients transmit $\mathrm{HCV}$ infection and contain replication competent viral RNA in complex with Ago2-miR122-HSP90. PLoS Pathog. 2014;10(10):e1004424. 